\title{
Proteomics characterization of novel spore proteins of Bacillus subtilis
}

\author{
Ritsuko Kuwana, ${ }^{1}$ Yasuhiro Kasahara, ${ }^{2}$ Machiko Fujibayashi, ${ }^{1}$ \\ Hiromu Takamatsu, ${ }^{1}$ Naotake Ogasawara ${ }^{2}$ and Kazuhito Watabe ${ }^{1}$
}

Author for correspondence: Kazuhito Watabe. Tel: +8172866 3112 or 3114 . Fax: +8172866 3112 or 3114 . e-mail:watabe@pharm.setsunan.ac.jp

\footnotetext{
1 Faculty of Pharmaceutical Sciences, Setsunan University, Hirakata, Osaka 573-0101, Japan

2 Nara Institute of Science and Technology, Ikoma, Nara 630-0101, Japan
}

\begin{abstract}
The spores of Bacillus subtilis have characteristic properties and consist of complex structures including various types of proteins. To perform comprehensive analysis of the protein composition of the spores, the proteins extracted from the spore were analysed by a combination of one-dimensional PAGE and liquid chromatography coupled to tandem mass spectrometry (LCMS/MS) using Turboquest SEQUEST software interfaced with the DNA sequence database of B. subtilis. A total of 154 proteins were identified, and 69 of them were novel. The remaining 85 proteins have been previously reported as sporulation-specific proteins or as proteins that are synthesized in vegetative cells. The expression pattern of each gene deduced to encode novel spore proteins was analysed using a series of strains carrying a lacz reporter gene. The results revealed that the expression of $\mathbf{2 6}$ genes was dependent on sporulation-specific sigma factors, namely $\sigma^{\mathrm{F}}, \sigma^{\mathrm{E}}, \sigma^{\mathrm{G}}$ and $\sigma^{\mathrm{K}}$. In this study, it is demonstrated that the combination of the techniques of SDS-PAGE and LCMS/MS, with the mutant library of $B$. subtilis, is an effective tool for the analysis of complicated cellular structures.
\end{abstract}

Keyword: mass spectrometry

\section{INTRODUCTION}

Bacterial sporulation is a relatively simple model for cell differentiation and its progress is marked by sequential and drastic changes in the physiological state of the cell. When nutrients are exhausted, the Gram-positive soil micro-organism Bacillus subtilis initiates sporulation by dividing asymmetrically. After asymmetric septation, the resultant larger and smaller cells are termed the mother cell and the forespore, respectively. As development proceeds, the mother cell engulfs the forespore and eventually lyses, releasing the mature spores into the medium. The mature spores can remain in the dormant stage for long periods of time and are resistant to heat, toxic chemicals, lytic enzymes and other factors that cause cell damage (Aronson \& Fitz-James, 1976; Goldman \& Tipper, 1978; Gould, 1983; Driks, 1999, 2001; Henriques \& Moran, 2000). Dormant spores respond rapidly to nutrients in the environment and return to an active physiological state through the process of germination (Paidhungat \& Setlow, 2001).

Abbreviation: LC-MS/MS, liquid chromatography coupled to tandem mass spectrometry.
Genes involved in the spore developmental system of $B$. subtilis have been identified and their biological functions have been analysed (Stragier \& Losick, 1996). These genes are mostly transcribed during sporulation by an RNA polymerase containing developmentally specific sigma factors, namely $\sigma^{\mathrm{F}}, \sigma^{\mathrm{E}}, \sigma^{\mathrm{G}}$ and $\sigma^{\mathrm{K}}$. These factors, forming a sigma cascade, are temporally and spatially activated and regulate gene expression in a compartment-specific fashion (reviewed by Piggot \& Losick, 2001). In the sigma cascade, $\sigma^{\mathrm{F}}$ is the primary sigma factor existing in the prespore and is required for the activation of pro- $\sigma^{\mathrm{E}}$ in the mother-cell compartment. Mature $\sigma^{\mathrm{E}}$ is required for the activation of $\sigma^{\mathrm{G}}$ in the forespore, and $\sigma^{\mathrm{G}}$ is required for the activation of pro- $\sigma^{\mathrm{K}}$ in the mother-cell compartment.

B. subtilis spores have three distinct structures, which can be observed using transmission electron microscopy (Aronson \& Fitz-James, 1976). The central part of the spore, called the core, includes chromosomal DNA. Some proteins required for protection of nucleotides and for the processes of germination and outgrowth are thought to exist in the core. The cortex is a thin peptidoglycan layer outside of the core that includes some proteins involved in germination. The outermost 
Table 1. Bacterial strains and plasmids used in this study

We obtained the B. subtilis strains, used for the LacZ assay shown in Fig. 2, from the Japanese and European Consortia for Functional Analysis of the B. subtilis Genome (respectively, http://bacillus.genome.ad.jp/ and http://genolist.pasteur.fr/).

\begin{tabular}{|c|c|c|}
\hline Strain/plasmid & Genotype/description & Source/reference \\
\hline \multicolumn{3}{|l|}{ Strain } \\
\hline B. subtilis 168 & $\operatorname{trp} C 2$ & 1A1 (Bacillus Genetic Stock Center) \\
\hline B. subtilis SGF602C & $\operatorname{trp} C 2 \operatorname{sig} F:: c a t$ & This work \\
\hline B. subtilis SGE603C & $\operatorname{trp} C 2$ sigE: :cat & This work \\
\hline B. subtilis SGG604C & $\operatorname{trp} C 2 \operatorname{sig} G:: c a t$ & This work \\
\hline B. subtilis SGK605C & $\operatorname{trpC2} \operatorname{spoIVCB}(\operatorname{sig} K):: c a t$ & This work \\
\hline E. coli JM109 & $\begin{array}{l}\text { relA supF44 endA1 hsdR17 gyrA96 morA mcrB } B^{+} \Delta \text { thi }\left(\text { lac-proAB) } / \mathrm{F}^{\prime}\right. \\
\quad\left(\text { traD36 proAB } \text { lacl }^{\alpha} \text { lacZ } \Delta \mathrm{M} 15\right)\end{array}$ & Sambrook et al. (1989) \\
\hline \multicolumn{3}{|l|}{ Plasmid } \\
\hline pUC18 & bla & Sambrook et al. (1989) \\
\hline pDH88 & bla cat spac-1 promoter lacI & Henner (1990) \\
\hline pCAT5 & cat & This work \\
\hline pSIGF62C & cat sigF $F^{\prime}$ & This work \\
\hline pSIGE63C & cat sigE & This work \\
\hline pSIGG64C & cat $\operatorname{sig} G^{\prime}$ & This work \\
\hline pSIGK65C & cat spoIVCB $(\operatorname{sig} K)^{\prime}$ & This work \\
\hline
\end{tabular}

layer is called the spore coat and is composed of two layers, the inner coat and the outer coat. The spore coat is mainly composed of variously sized proteins, some of which function in cell development and morphogenesis; however, a limited number of them have been characterized because of the difficulty in their isolation or purification by conventional procedures (Driks, 2001). Therefore, the complete composition of sporeassociated proteins and their characteristics are still unknown.

The $B$. subtilis genome-sequencing project revealed about 4100 protein-encoding genes, half of which have unknown functions (Kunst et al., 1997). Systematic disruption of the remaining genes has already been carried out by the Japanese and European Consortia for Functional Analysis of the B. subtilis Genome (respectively, http://bacillus.genome.ad.jp/ and http:// genolist.pasteur.fr/). The strategy for construction of the mutants was insertional inactivation of the target genes with pMutin carrying a lac Z reporter gene (Vagner et al., 1998). Recently, almost all of the disruptant strains of $B$. subtilis have been made available for analysis.

The recent abundance of genome sequence data has brought an urgent need for the use of systematic proteomics to gain an understanding of the protein network that directs cellular functions, especially the sporulation, germination and spore dormancy of bacilli. Liquid chromatography coupled to tandem mass spectrometry (LC-MS/MS) is a powerful technique for the analysis of peptides and proteins and is now widely applied to proteome analysis. Multiprotein complexes function in the processes of bacterial sporulation and germination as well as most other cellular processes in many organisms. Here, we applied LC-MS/MS to a comprehensive analysis of proteins extractable from whole spores of $B$. subtilis, and 154 proteins were found. We also analysed the regulation of genes encoding each spore protein using cells with mutations in genes for sporulation-specific sigma factors.

\section{METHODS}

Bacterial strains, plasmids, media and general techniques. The B. subtilis and Escherichia coli strains used in this study are listed in Table 1. B. subtilis 168 (trpC2) (BGSC 1A1), obtained from the Bacillus Genetic Stock Center (Ohio State University, OH, USA), was used in this study as a wild-type strain. We also used a series of strains that have been constructed by the Japanese and European Consortia for Functional Analysis of the B. subtilis Genome (respectively, http://bacillus.genome.ad.jp/ and http://genolist.pasteur. $\mathrm{fr} /)$. The unified method for the construction of these strains has been described previously; in each strain, pMutin carrying a lac $Z$ reporter gene is inserted into an appropriate position within the genome to inactivate a target gene (Vagner et al., 1998). Oligonucleotide primers CATM172 (5'-TCTAGTACTAAAGCACCCATTAGTTC-3'), CAT686R (5'-TTAGATATCCGACTGTAAAAAGTACAGT-3'), PUC1697 (5'-AAAGATATCTAGGTGAAGATCCTTTTT-3') and PUC723R (5'-TTAAGTACTCATGAGCGGATACATATT$\left.3^{\prime}\right)$ were used to amplify a $515 \mathrm{bp}$ segment of a chloramphenicol-resistance gene (cat) of pDH88 (Henner, 1990) and a 974 bp segment of pUC18 (Sambrook et al., 1989) including the origin of replication and the multiple cloning site. The PCR products were digested at the EcoRV and ScaI sites, and the resultant DNA fragments were ligated to create pCAT5. Oligonucleotide primers SIGF2428 (5'-TAAAAGCTTCGGCAAAAACGCTCAGCT-3') and SIGF207R (5'-TTAGGATCCCGATGCAGCCGATCT-3') were used to amplify a 183 bp segment internal to sigF on the B. subtilis 168 


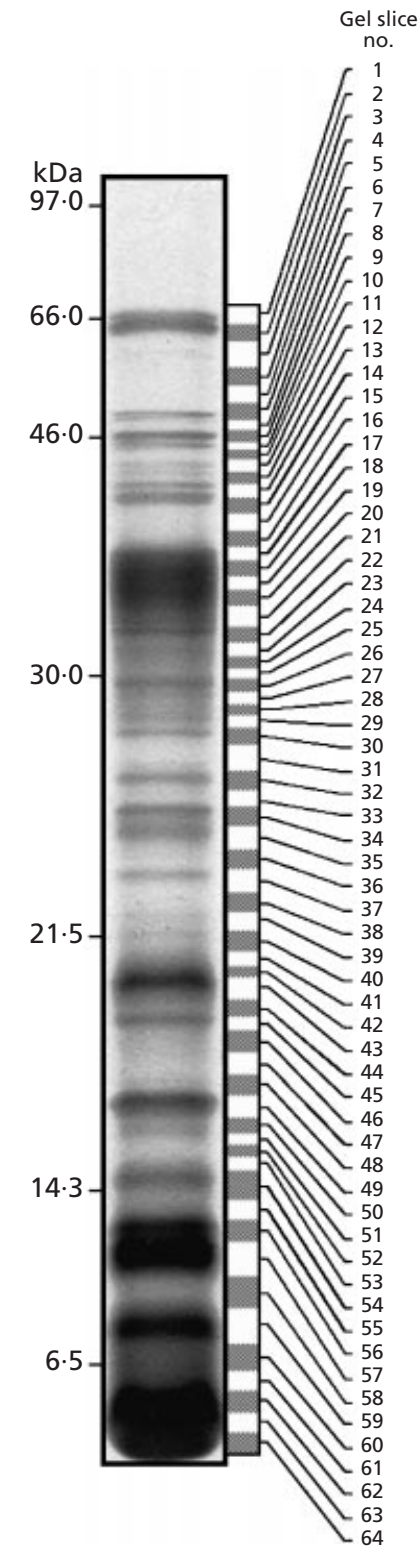

Fig. 1. SDS-PAGE was used to resolve the protein extracts from $B$. subtilis spores. Proteins were extracted from mature spores of $B$. subtilis in the presence of SDS and mercaptoethanol, as described in Methods. The protein preparation (approx. $20 \mu \mathrm{g}$ ) was resolved by SDS-PAGE ( $14 \%, w / v$, acrylamide gel) and the proteins were stained with Coomassie brilliant blue. The gel was then divided into 64 slices and analysed by LC-MS/MS.

chromosome. The PCR product was digested at the BamHI and HindIII sites introduced by the primers and then inserted into BamHI- and HindIII-restricted pCAT5 to create pSIGF62C. Oligonucleotide primers SIGE16 (5'-ACTAAGCTTACGGTTGACGCACCTC-3') and SIGE208R (5'-TATGGATCCAGACGCAAAATGCGTTC-3') were used to amplify a 192 bp segment internal to sigE on the B. subtilis 168 chromosome. The PCR product was digested at the BamHI and HindIII sites introduced by the primers and then inserted into BamHI- and HindIII-restricted pCAT5 to create pSIGE63C. Oligonucleotide primers SIGG121 (5'-GAAAA-
GCTTGTAAACGGGAACTT-3') and SIGG315R (5'-GTAGGATCCTGCGGATCTCTCCGA-3') were used to amplify a $194 \mathrm{bp}$ segment internal to sigG on the B. subtilis 168 chromosome. The PCR product was digested at the Bam HI and HindIII sites introduced by the primers and then inserted into BamHI- and HindIII-restricted pCAT5 to create pSIGG64C. Oligonucleotide primers SIGK16 (5'-AGGAAGCTTCGCAGCGCTCGGCTT-3') and SIGK228R (5'-TCGCTCGAGAAGTTACCATTGTGGATTC-3') were used to amplify a 212 bp segment internal to spoIVCB (sigK) from the B. subtilis 168 chromosome. The PCR product was digested at the BamHI and HindIII sites introduced by the primers and then inserted into BamHI- and HindIII-restricted pCAT5 to create pSIGK65C. Plasmids pSIGF62C, pSIGE63C, pSIGG64C and pSIGK65C were introduced into B. subtilis 168 under selection for chloramphenicol resistance (5 $\mathrm{g}$ chloramphenicol ml $\mathrm{ml}^{-1}$ ) to produce the transformants SGF602C, SGE603C, SGG604C and SGK605C, respectively. The plasmids were integrated into the chromosomal DNA by homologous recombination and the resultant transformants were tested by PCR. The chromosomal DNA of pMutin strains was introduced into SGF602C, SGE603C, SGG604C or SGK605C to construct double mutants of a sigma factor gene and each gene tested in this study. The resultant cells were resistant to both erythromycin and chloramphenicol. $B$. subtilis strains were grown in Difco Sporulation (DS) medium; E. coli was grown in Luria-Bertani (LB) medium (Takamatsu et al., 2000). The conditions for sporulation of B. subtilis have been described previously (Takamatsu et al., 2000). Recombinant DNA techniques were carried out following standard protocols (Sambrook et al., 1989). Methods for preparing competent cells, for transformation and for the preparation of chromosomal DNA of B. subtilis have been described previously (Cutting \& Vander Horn, 1990).

Preparation of spores. The wild-type strain of B. subtilis (168) was grown in DS medium at $37^{\circ} \mathrm{C}$ as described previously, and mature spores were harvested $18 \mathrm{~h}$ after the cessation of exponential growth $\left(T_{18}\right)$ and washed once with $10 \mathrm{mM}$ sodium phosphate buffer (pH 7·2) (Takamatsu et al., 2000). To remove the cell debris and vegetative cells, the pellets were suspended in $0.1 \mathrm{ml}$ lysozyme buffer $[10 \mathrm{mM}$ sodium phosphate $(\mathrm{pH} 7 \cdot 2), 1 \%(\mathrm{w} / \mathrm{v})$ lysozyme, Complete protease inhibitor cocktail (Roche)] and incubated at room temperature for $10 \mathrm{~min}$. They were then washed repeatedly with buffer [10 mM sodium phosphate $(\mathrm{pH} 7 \cdot 2), 0.5 \mathrm{M} \mathrm{NaCl}]$ at room temperature (Takamatsu et al., 2000). After these treatments, more than $99.9 \%$ of the spores were refractile and few dark spores were visible under the phase-contrast microscope. Neither vegetative cells nor cell debris were included in the sample (data not shown).

Solubilization of proteins from mature spores for SDS-PAGE. Spore proteins were solubilized in $0.1 \mathrm{ml}$ loading buffer [62.5 mM Tris/HCl (pH 6.8), 4\% (w/v) SDS, $10 \%$ (v/v) 2mercaptoethanol, $10 \%$ (v/v) glycerol, $0.05 \%(\mathrm{w} / \mathrm{v})$ bromophenol blue] and boiled for $5 \mathrm{~min}$ as described previously (Takamatsu et al., 2000). The proteins were separated by $14 \%$ SDS-PAGE and visualized by Coomassie brilliant blue R-250 staining (Takamatsu et al., 2000).

In-gel enzymic digestion. Coomassie-blue-stained bands were serially divided into 64 slices from the top band $(66 \mathrm{kDa})$ to the bromophenol blue line at the bottom of the gel at intervals of about $1 \mathrm{~mm}$. The gel slices were washed, reduced by DTT, alkylated by iodoacetamide and digested with modified trypsin or chymotrypsin (Roche) at $37^{\circ} \mathrm{C}$ for $16 \mathrm{~h}$ as described previously (Shevchenko et al., 1996). The resultant peptides 
Table 2. Proteins identified in extracts from $B$. subtilis spores

The 64 gel slices described in Fig. 1 were analysed by LC-MS/MS; the proteins detected in each slice are listed alphabetically.

\begin{tabular}{|c|c|c|c|c|c|}
\hline $\begin{array}{l}\text { Gel slice } \\
\text { no. }\end{array}$ & $\begin{array}{l}\text { Molecular } \\
\text { mass }(\mathrm{kDa})\end{array}$ & Detected protein & $\begin{array}{l}\text { Gel slice } \\
\text { no. }\end{array}$ & $\begin{array}{l}\text { Molecular } \\
\text { mass }(\mathrm{kDa})\end{array}$ & Detected protein \\
\hline 1 & 66 & CotA CotB Epr & 33 & $25 \cdot 5$ & CotE SodA SqhC YcsK YlaJ YrbB \\
\hline 2 & 63 & CotA CotB DppE & 34 & 25 & CotE CotJC CotY YhcN YlaJ YrbB \\
\hline 3 & 59 & CotA CotB CotE OppA YcgN YuiE & 35 & $24 \cdot 5$ & CotE CotJC GltA YhcN YrbB \\
\hline 4 & 55 & CotA CotB CotE YcgN & 36 & $23 \cdot 5$ & CotE YwdL YxeE \\
\hline 5 & 52 & CotB CotE CotY YaaH YdbR YopK & 37 & 23 & CotE CotY YwdL YxeE \\
\hline 6 & 50 & CotB YaaH & 38 & $22 \cdot 5$ & CotE CotD CotY \\
\hline 7 & 48 & $\begin{array}{l}\text { CotB CotE CotY MelA YaaH YpeB } \\
\text { YvdP }\end{array}$ & 39 & 22 & CotE CotY YhcM YxkC \\
\hline 8 & 46 & CotB PhoA TufA YgaK YtxN YvdP & 40 & $21 \cdot 5$ & CotE CotY YdcN \\
\hline 9 & 45 & $\begin{array}{l}\text { AhpF CotE PhoA YdhO YgaK YtxN } \\
\text { YvdP }\end{array}$ & 41 & $20 \cdot 5$ & CotE CotY YqaN YwqH YxeE \\
\hline 10 & 44 & $\begin{array}{l}\text { CotB CotY YdhD YhcY YtcC YtxN } \\
\text { YvrK }\end{array}$ & 42 & 20 & CotC CotE CotY YhcM YnzH \\
\hline 11 & $43 \cdot 5$ & CotB Eno YdhD YkvP & 43 & $19 \cdot 5$ & $\begin{array}{l}\text { CotC CotE CotY GerD SpoIVA YnzH } \\
\text { YxeE }\end{array}$ \\
\hline 12 & 43 & $\begin{array}{l}\text { CotB CotE DacF YhfE YjbX YopG } \\
\text { YtaA }\end{array}$ & 44 & 19 & $\begin{array}{l}\text { CotE CotY QcrA RpsG SpoIVA YnzH } \\
\text { YxeE }\end{array}$ \\
\hline 13 & 42 & $\begin{array}{l}\text { AprX CotB CotE CotH CotS DacF } \\
\text { YheC YoaN YtaA }\end{array}$ & 45 & $18 \cdot 5$ & $\begin{array}{l}\text { CgeA CotC CotD CotE CotY CwlJ } \\
\text { FtsH RpsG RpsL SpoIVA }\end{array}$ \\
\hline 14 & 41 & CotB CotS Gap YoaN & 46 & 18 & $\begin{array}{l}\text { CotC CotD CotE CotY CotZ CwlJ } \\
\text { RpsL YkuD YopY YtfJ YxeE }\end{array}$ \\
\hline 15 & 39 & CotB CotE DacB YoaN & 47 & $17 \cdot 5$ & $\begin{array}{l}\text { CotC CotD CotE CotY SspE YdjK } \\
\text { YjfA YvdP }\end{array}$ \\
\hline 16 & 38 & CotB CotE CotG CotY YufD & 48 & 17 & CotC CotE CotD SspE YjcN YrkC \\
\hline 17 & 37 & $\begin{array}{l}\text { CotB CotE CotG CitH CotY YerC } \\
\text { YhxC }\end{array}$ & 49 & $16 \cdot 5$ & CotC CotD CotE SspE YrkC \\
\hline 18 & 36 & CotE CotG & 50 & 16 & CotC CotD CotE SspE YhcV YhjR \\
\hline 19 & 35 & CotG CotY YtdI & 51 & $15 \cdot 5$ & $\begin{array}{l}\text { CotC CotD CotE CotS PksM RplR } \\
\text { RplX SspE YhjR }\end{array}$ \\
\hline 20 & 34 & $\operatorname{Cot} B \operatorname{Cot} E \operatorname{Cot} G \operatorname{Cot} Y$ & 52 & 15 & $\begin{array}{l}\text { CotC CotD CotE CotS RbfA RplQ } \\
\text { RplR RplX YhjR }\end{array}$ \\
\hline 21 & 33 & $\begin{array}{l}\text { CotB CotE CotG PrsA YdjK YqfQ } \\
\text { YuxL YvdO }\end{array}$ & 53 & $14 \cdot 5$ & CotD CotE CotW CotY \\
\hline 22 & 32 & $\begin{array}{l}\text { CotB CotG CtaC CysK Gdh PrsA } \\
\text { YfmC YvdO }\end{array}$ & 54 & 14 & CotD CotW YfhD YqjR \\
\hline 23 & 31 & CotB CotE CotG YjqC YusA YvdO & 55 & $13 \cdot 5$ & $\begin{array}{l}\text { CotB CotD CotE CotW DnaE RpmA } \\
\text { Tlp YqaN YrdD }\end{array}$ \\
\hline 24 & $30 \cdot 5$ & CotB CotE CotG YhcH YjqC YusA & 56 & 12 & CotB CotC CotE RpmA \\
\hline 25 & 30 & CotE CotG SleB YisY YvdO & 57 & 11 & $\begin{array}{l}\text { CotB CotC CotD CotE YabG YfhD } \\
\text { YhfS }\end{array}$ \\
\hline 26 & $29 \cdot 5$ & $\begin{array}{l}\text { CotE CotG SleB YcdA YckK YisY } \\
\text { YitS YtxO YvdO }\end{array}$ & 58 & $9 \cdot 5$ & CotB CotC CotD RpmI YxeE \\
\hline 27 & 29 & CotE LipA & 59 & 8 & CotC CotD RpsP YdbO YfjU YxeE \\
\hline 28 & $28 \cdot 5$ & $\begin{array}{l}\text { CotD CotE DnaE RplA RpsC SpsI } \\
\text { YfkD YqiX YrbA YvgV YwrJ }\end{array}$ & 60 & $6 \cdot 5$ & CotD Hbs RpsP YjdH YkzE YxeE \\
\hline 29 & 28 & CotE Tgl YhjI YvdD YybI & 61 & 6 & $\begin{array}{l}\text { CotB CotD CotE CotJA RpsO RpsT } \\
\text { YjdH YkzE YuzC YxeE }\end{array}$ \\
\hline 30 & $27 \cdot 5$ & CotE YbaN YdcC YerQ & 62 & $5 \cdot 5$ & CotC CotD CotE CotF YsnD \\
\hline 31 & 27 & CotE Mpr YdcC YvaB & 63 & 5 & $\begin{array}{l}\text { CotD CotF CotJB CotK CotL SspC } \\
\text { YclI YodI YkzE YmaG }\end{array}$ \\
\hline 32 & 26 & CotE Mpr SodF YcsK YdcC & 64 & $<4 \cdot 5$ & $\begin{array}{l}\text { CotD CotJB CotK RpmC SspB SspC } \\
\text { YmaG YodI }\end{array}$ \\
\hline
\end{tabular}


were eluted from the gel with several changes of extraction buffer [70 $\mu \mathrm{l}$ of $70 \%(\mathrm{v} / \mathrm{v})$ acetonitrile, $5 \%(\mathrm{v} / \mathrm{v})$ formic acid] and concentrated by evaporation. The samples were then diluted with $5 \%$ formic acid and $50 \%$ acetonitrile for further analysis.

Peptide analysis and protein identification by LC-MS/MS. Nanoliquid chromatography combined with nanoelectrospray ion-trapping tandem mass spectrometry (nanoLCnanoESI-MS/MS) analysis of peptides was carried out with an LC-Q Deca mass spectrometer (Thermoquest) coupled with a microcapillary nano-flow LC Magic 2002 (Michrom Bioresources). Protein identification was performed using the SEQUEST search program (Thermoquest) (Yates et al., 1995). The searched database was the Bacillus subtilis Protein Database.

LacZ assay for evaluation of gene expression. The Japanese and European Consortia for Functional Analysis of the $B$. subtilis Genome constructed the pMutin strains that we used in this study. Each strain contains a lacZ fusion protein to monitor gene expression (Vagner et al., 1998). Chromosomal DNA from the strains was extracted and introduced into the sigma-defective mutants SGF602C, SGE603C, SGG604C and SGK605C by competent cell transformation. Resultant cells were grown on DS sporulation agar medium including $\mathrm{X}-\mathrm{Gal}$ for $48 \mathrm{~h}$ at $37^{\circ} \mathrm{C}$ and the colony colour was monitored to detect expression of each gene. Five independent colonies of each transformant were tested.

\section{RESULTS}

\section{Analysis of spore proteins by SDS-PAGE and LC- MS/MS}

The proteins solubilized from dormant spores in this study were first resolved by SDS-PAGE and the gel was divided into 64 slices from the position of the $66 \mathrm{kDa}$ band to the bottom line $(4.5 \mathrm{kDa})$ as shown in Fig. 1 . Each gel slice in-gel-digested was then analysed by LCMS/MS. The MS/MS raw data were used to search with the SEQUEST search program. To identify protein, generally, a correlation factor (Xcorr) greater than $2 \cdot 0$ indicates a highly significant match, and a delta crosscorrelation factor $(\mathrm{DelC} n)$ higher than $0 \cdot 1$ indicates a significant distinction between the best match and the second-best match. From search results, the proteins detected in each gel slice are summarized in Table 2. A total of 154 proteins were identified. The predicted characteristics of 85 proteins are described in the $B$. subtilis Genome Database (http://bacillus.genome. ad.jp/). Of these proteins, 41 have been previously reported as sporulation-specific proteins, and another 44 proteins have been previously reported to be synthesized in vegetatively growing cells or sporangia (Table 3 ). The remaining 69 proteins identified here have not been reported previously (Table 4). The molecular masses of the proteins identified from gels mostly coincided with the deduced molecular masses in the database. However, some proteins, such as the spore-coat proteins CotB, $\operatorname{Cot} \mathrm{C}, \operatorname{Cot} \mathrm{D}, \operatorname{Cot} \mathrm{E}, \operatorname{Cot} \mathrm{S}$ and $\operatorname{Cot} \mathrm{Y}$, were dispersed among several gel slices and their sizes were calculated based on the range of molecular masses estimated for each protein from the SDS-PAGE analysis.

\section{Expression patterns of genes encoding the proteins extracted from mature spores}

To characterize B. subtilis genes of unknown function, a series of strains have been constructed using derivatives of a plasmid, pMutin, by the Japanese and European Consortia for Functional Analysis of the B. subtilis Genome [JAFAN (http://bacillus.genome.ad.jp/) and SubtiList (http://genolist.pasteur.fr/), respectively]. These plasmids have a lac $Z$ reporter gene (Vagner et al., 1998). In each pMutin strain, lacZ is located downstream of the $5^{\prime}$ portion of a target gene and becomes transcriptionally active when the target gene is activated. The strains provided by the Consortia allow the pattern of target gene expression to be monitored. In this study, we tried to identify sporulation-specific genes encoding the proteins extracted from $B$. subtilis spores. Two different methods are available for monitoring of $\mathrm{LacZ}$ activity; one is an enzyme assay on cells grown in liquid medium and the other is a plate colony assay, using X$\mathrm{Gal}$ as a chromogenic substrate. We preferred the plate colony assay, because this method can be advantageous for performing systematic and first-step screening of the genes involved in sporulation. Of the 69 genes known to be involved in sporulation, we could analyse 57; we excluded the remaining 12 genes from our study for the following reasons. Nine genes, $y d c N, y k z E, y n z H$, yodI, yopG, yopK, yopY, yqaN and $y r d D$, were not available from the Consortia stocks. These genes either originated from phages or the construction of disruptant strains is difficult. The three genes $y e r Q, y j c N$ and $y u f D$ are essential or are related to essential genes for growth. Further analysis of these genes was not necessary, because the purpose of this work was to identify genes specifically transcribed during sporulation. We examined 57 strains and found that 48 expressed LacZ activity on sporulation medium (Fig. 2). The remaining nine strains did not show any LacZ activity (data not shown), suggesting that the expression of $y c l l, y g a K$, $y h c Y, y i t S, y j b X, y j q C, y q j R, y v d O$ and $y y b I$ was too weak to be detected under our experimental conditions. The 48 genes that were possibly expressed during sporulation were then further studied as described below.

The expression of the 48 genes possibly expressed during sporulation was analysed by monitoring LacZ activity in sigma-factor-defective cells, such as SigF, SigE, SigG or SigK (Fig. 2, lanes F, E, G and K, respectively). These factors are sporulation-specific and are activated sequentially during sporulation (Driks, 2001; Helmann \& Moran, 2001). Each chromosomal DNA of the pMutin strains was transformed into each sigma mutant. The resultant transformants were grown on sporulation medium and their LacZ activity was monitored. The expression of 26 of the 48 genes was controlled by the 
Table 3. List of previously identified proteins that were found to be associated with wild-type spores

Among those extracted from mature spores of B. subtilis, the proteins whose characteristics have been reported are listed alphabetically. Some of them have been renamed already. The current names and ORF IDs are indicated according to the Bacillus subtilis Genome Database JAFAN (http://bacillus.genome.ad.jp). The molecular masses of the proteins were deduced from amino acid sequence data and by the migration positions on the acrylamide gel shown in Fig. 1. The molecular masses of the proteins detected in gel slice no. 64 were estimated to be smaller than $6.4 \mathrm{kDa}$. Possible signal sequences and the number of transmembrane sequences are based upon information from the JAFAN database.

\begin{tabular}{|c|c|c|c|c|c|}
\hline \multirow{2}{*}{$\begin{array}{l}\text { Protein } \\
\text { name }\end{array}$} & \multirow{2}{*}{$\begin{array}{l}\text { ORF } \\
\text { ID }\end{array}$} & \multicolumn{2}{|c|}{ Molecular mass (kDa) } & \multirow{2}{*}{$\begin{array}{l}\text { Localization } \\
\text { signal }\end{array}$} & \multirow[t]{2}{*}{ Description } \\
\hline & & $\begin{array}{l}\text { Deduced from } \\
\text { sequence }\end{array}$ & $\begin{array}{l}\text { Calculated by } \\
\text { SDS-PAGE }\end{array}$ & & \\
\hline AhpF & BG11204 & 55 & 44 & & Alkyl hydroperoxide reductase \\
\hline AprX & BG12567 & 48 & 42 & & Alkaline serine protease \\
\hline CgeA & BG11193 & 14 & 19 & & Involved in spore-surface property \\
\hline $\mathrm{CitH}$ & BG11146 & 34 & 37 & Signal sequence & Malate dehydrogenase \\
\hline $\operatorname{Cot} \mathrm{A}$ & BG10490 & 58 & $66,63,59,55$ & & Spore-coat protein \\
\hline CotB & BG10491 & 43 & $66-6 \cdot 0$ & & Spore-coat protein \\
\hline CotC & BG10492 & 15 & $20-5 \cdot 5$ & & Spore-coat protein \\
\hline CotD & BG10493 & $8 \cdot 8$ & 29 to $<4.5$ & & Spore-coat protein \\
\hline CotE & BG10494 & 21 & $59-5 \cdot 5$ & & Involved in coat assembly \\
\hline $\operatorname{CotF}$ & BG10012 & 19 & $5 \cdot 5,5 \cdot 0$ & & Spore-coat protein \\
\hline CotG & BG11017 & 24 & 37 to $<4.5$ & & Spore-coat protein \\
\hline $\mathrm{CotH}$ & BG11791 & 43 & 42 & & Involved in coat assembly \\
\hline CotJA & BG11799 & $9 \cdot 7$ & $6 \cdot 0,5 \cdot 0$ & & Spore-coat protein \\
\hline CotJB & BG11800 & 12 & $<4 \cdot 5$ & & Spore-coat protein \\
\hline CotJC & BG11801 & 22 & 25 & & Spore-coat protein \\
\hline CotK $(\mathrm{SspO})$ & BG11920 & $5 \cdot 4$ & $5 \cdot 0,<4 \cdot 5$ & & Small acid-soluble spore protein \\
\hline $\operatorname{CotL}(\mathrm{SspP})$ & BG11921 & $5 \cdot 4$ & 5 & & Small acid-soluble spore protein \\
\hline CotS & BG11380 & 41 & $42,41,16,15$ & & Spore-coat protein \\
\hline CotW & BG10497 & 12 & 15,14 & & Spore-coat protein \\
\hline $\operatorname{Cot} Y$ & BG10498 & 18 & $52-15$ & & Spore-coat protein \\
\hline CotZ & BG10499 & 17 & 18 & & Spore-coat protein \\
\hline $\mathrm{CtaC}$ & BG10215 & 40 & 32 & Transmembrane (3) & Cytochrome $c$ oxidase I \\
\hline CwlJ & BG11172 & 16 & 19,18 & & Germination protein \\
\hline CysK & BG10136 & 33 & 32 & Transmembrane (1) & Cysteine synthase \\
\hline $\mathrm{DacB}$ & BG10527 & 43 & 39 & Signal sequence & Involved in cortex development \\
\hline $\mathrm{DacF}$ & BG10295 & 43 & 43 & Signal sequence & Involved in cortex development \\
\hline DnaE & BG12583 & 125 & 29,14 & & DNA polymerase III \\
\hline DppE & BG10846 & 63 & 63 & Signal sequence & Dipeptide ABC transporter \\
\hline Eno & BG10899 & 47 & 44 & & Enolase \\
\hline Epr & BG10561 & 70 & 66 & Signal sequence & Serine protease \\
\hline FtsH & BG10132 & 71 & 19 & Transmembrane (2) & Membrane metalloprotease \\
\hline Gap & BG10827 & 36 & 41 & & Glucose dehydrogenase in core \\
\hline GDH & BG10545 & 28 & 32 & & Glyceraldehyde-3-phosphate dehydrogenase \\
\hline GerD & BG10644 & 21 & 20 & Signal sequence & Germination protein \\
\hline GltA & BG10811 & 17 & 25 & & Glutamate synthase \\
\hline Hbs & BG10276 & $9 \cdot 9$ & $6 \cdot 5$ & & Non-specific DNA-binding protein \\
\hline LipA & BG14038 & 23 & 31 & Signal sequence & Lipase \\
\hline MelA & BG12615 & 49 & 48 & & $\alpha$-D-Galactoside galactohydrolase \\
\hline Mpr & BG10690 & 34 & 27,26 & Signal sequence & Extracellular metalloprotease \\
\hline OppA & BG10771 & 61 & 59 & Signal sequence & Oligopeptide ABC transporter \\
\hline PhoA & BG10183 & 50 & 46,45 & Signal sequence & Alkaline phosphatase A \\
\hline PksM & BG10931 & 477 & 16 & & Polyketide synthase \\
\hline PrsA & BG10464 & 32 & 33,32 & Signal sequence & Protein secretion \\
\hline QcrA & BG11325 & 19 & 19 & Signal sequence & Menaquinol cytochrome $c$ oxidase \\
\hline RbfA & BG10270 & 13 & 15 & & Ribosome-binding factor A \\
\hline RplA & BG10164 & 25 & 29 & & Ribosomal protein L1 \\
\hline RplQ & BG11041 & 14 & 15 & & Ribosomal protein L17 \\
\hline
\end{tabular}


Table 3. (cont.)

\begin{tabular}{|c|c|c|c|c|c|}
\hline \multirow{2}{*}{$\begin{array}{l}\text { Protein } \\
\text { name }\end{array}$} & \multirow{2}{*}{$\begin{array}{l}\text { ORF } \\
\text { ID }\end{array}$} & \multicolumn{2}{|c|}{ Molecular mass $(\mathrm{kDa})$} & \multirow{2}{*}{$\begin{array}{l}\text { Localization } \\
\text { signal }\end{array}$} & \multirow{2}{*}{ Description } \\
\hline & & $\begin{array}{l}\text { Deduced from } \\
\text { sequence }\end{array}$ & $\begin{array}{l}\text { Calculated by } \\
\text { SDS-PAGE }\end{array}$ & & \\
\hline RplR & BG11409 & 13 & 16,15 & & Ribosomal protein L18 \\
\hline RplX & BG10759 & 11 & 16,15 & & Ribosomal protein L24 \\
\hline RpmA & BG10335 & 10 & 13,12 & & Ribosomal protein L27 \\
\hline $\mathrm{RpmC}$ & $B G 10756$ & $7 \cdot 7$ & $<4 \cdot 5$ & & Ribosomal protein L29 \\
\hline RpmI & BG11972 & $7 \cdot 6$ & $9 \cdot 5$ & & Ribosomal protein L35 \\
\hline $\mathrm{RpsC}$ & BG19005 & 24 & 29 & & Ribosomal protein S3 \\
\hline RpsG & BG19006 & 18 & 19 & & Ribosomal protein S7 \\
\hline RpsL & BG19009 & 15 & 19,18 & & Ribosomal protein S12 \\
\hline $\mathrm{RpsO}$ & BG19010 & 11 & 6 & & Ribosomal protein S15 \\
\hline $\mathrm{RpsP}$ & BG10831 & 10 & $8 \cdot 0,6 \cdot 5$ & & Ribosomal protein S16 \\
\hline RpsT & BG11643 & $9 \cdot 5$ & $5 \cdot 5$ & & Ribosomal protein S20 \\
\hline SleB & BG11439 & 34 & 30 & Signal sequence & Germination protein in cortex \\
\hline SodA & $B G 11676$ & 25 & 26 & & Superoxide dismutase \\
\hline SodF & $B G 12676$ & 33 & 26 & & Superoxide dismutase \\
\hline SpoIVA & $B G 10275$ & 55 & 20,19 & & Involved in coat and cortex development \\
\hline SpsI & $B G 10617$ & 28 & 29 & & Spore-coat-polysaccharide synthesis \\
\hline SqhC & $B G 12679$ & 63 & 44,26 & & Squalene/hopene cyclase \\
\hline SspB & BG10787 & 7 & $<4 \cdot 5$ & & Small acid-soluble spore protein \\
\hline SspC & BG10882 & $7 \cdot 8$ & $5 \cdot 0,<4 \cdot 5$ & & Small acid-soluble spore protein \\
\hline SspE & BG10789 & $9 \cdot 3$ & $18,17,16$ & & Small acid-soluble spore protein \\
\hline Tgl & BG10946 & 28 & 28 & & Transglutaminase \\
\hline Tlp & BG11806 & $9 \cdot 7$ & 14,10 & & Thioredoxin-like protein \\
\hline TufA & BG11056 & 44 & 46 & & Elongation factor $\mathrm{Tu}$ \\
\hline $\mathrm{YaaH}$ & BG10080 & 49 & $52,50,48$ & $\begin{array}{l}\text { Cell-wall-binding } \\
\text { motif }\end{array}$ & Germination protein \\
\hline YabG & BG10106 & 33 & 11 & & Protease involved in coat assembly \\
\hline YbaN & $B G 11416$ & 28 & 28 & Signal sequence & Involved in sporulation \\
\hline YdhD & BG12181 & 49 & 44 & $\begin{array}{l}\text { Cell-wall-binding } \\
\text { motif }\end{array}$ & Spore protein involved in germination \\
\hline YdjK (IolT) & BG12802 & 52 & 33 & Transmembrane (11) & Major inositol-transport protein \\
\hline YfjU $(\mathrm{SspH})$ & BG12917 & $6 \cdot 9$ & 8 & & Small acid-soluble spore protein \\
\hline YhcN & BG11592 & 21 & 25 & Signal sequence & Involved in outgrowth \\
\hline YkuD & BG13288 & 18 & 18 & $\begin{array}{l}\text { Cell-wall-binding } \\
\text { motif }\end{array}$ & Spore protein \\
\hline YkvP & BG13318 & 46 & $43 \cdot 5$ & $\begin{array}{l}\text { Cell-wall-binding } \\
\text { motif }\end{array}$ & Spore protein \\
\hline YoaN & $B G 13484$ & 44 & $42,41,39$ & & Oxalate decarboxylase \\
\hline YpeB & BG11001 & 51 & 48 & Signal sequence & Germination protein \\
\hline YrbA (SafA) & BG13781 & 43 & 29 & $\begin{array}{l}\text { Cell-wall-binding } \\
\text { motif }\end{array}$ & Coat protein involved in coat assembly \\
\hline YrbB & $B G 13782$ & 20 & 26,25 & Signal sequence & Cortex protein \\
\hline $\begin{array}{l}\text { YtxN } \\
(\text { CotSA) }\end{array}$ & $B G 11381$ & 43 & $46,45,44$ & & Spore-coat protein \\
\hline YvrK & BG14148 & 44 & 44 & & Oxalate decarboxylase \\
\hline
\end{tabular}

sporulation-specific sigma factors. From the results shown in Fig. 2 and the known sigma cascade of $B$. subtilis, possible regulators of the novel spore proteins are summarized in Table 4 . The transcription of the $y f h D, y h c M$ and $y t f J$ genes in the prespore is possibly controlled by $\sigma^{\mathrm{F}}$. The transcription of the $y d c \mathrm{C}, y h e C$, $y h j R, y h x C, y j d H, y j f A, y s n D, y u z C, y w d L$ and $y x e E$ genes in the mother-cell compartment is possibly controlled by $\sigma^{\mathrm{E}}$. The transcription of the $y f k D, y h c V, y i s Y$, $y l a J$ and $y v d P$ genes in the forespore is possibly controlled by $\sigma^{\mathrm{G}}$. The transcription of $y c s K, y m a G$, $y q f Q, y r k C, y t a A, y t c C, y t x \mathrm{O}$ and $y w r J$ in the mother- 
Table 4. Description of the novel spore proteins identified in this study

Among those extracted from mature spores of B. subtilis, the proteins whose characteristics have not been reported are listed alphabetically. ORF IDs are according to the Bacillus subtilis Genome Database JAFAN (http://bacillus.genome.ad.jp). The molecular mass of each protein was deduced from amino acid sequence data and by the migration position on the acrylamide gel shown in Fig. 1. The molecular masses of the proteins detected in gel slice no. 64 were estimated to be smaller than $6.4 \mathrm{kDa}$. Possible signal sequences and the number of transmembrane sequences are based upon information from the JAFAN database. Predictions of the sigma factors that regulate the synthesis of each protein were based upon the results of the LacZ assay described in Fig. 2. SigA and/or other sigma factors possibly regulate the proteins whose syntheses are independent of SigF, SigE, SigG or SigK.

\begin{tabular}{|c|c|c|c|c|c|}
\hline \multirow[t]{2}{*}{ Protein name } & \multirow[t]{2}{*}{ ORF ID } & \multicolumn{2}{|c|}{ Molecular mass (kDa) } & \multirow[t]{2}{*}{ Localization signal } & \multirow[t]{2}{*}{ Possible regulator } \\
\hline & & $\begin{array}{l}\text { Deduced from } \\
\text { sequence }\end{array}$ & $\begin{array}{l}\text { Calculated by } \\
\text { SDS-PAGE }\end{array}$ & & \\
\hline YcdA & $B G 12757$ & 39 & 30 & Signal sequence & SigA and/or others \\
\hline $\mathrm{YcgN}$ & BG12012 & 56 & 59,55 & & SigA and/or others \\
\hline YckK & $B G 11187$ & 29 & 30 & Signal sequence & SigA and/or others \\
\hline YclI & BG12030 & 52 & $5 \cdot 0,<4 \cdot 5$ & Transmembrane (4) & $\mathrm{ND}$ \\
\hline $\mathrm{YcsK}$ & BG11232 & 24 & 26 & & SigK \\
\hline $\mathrm{YdbO}$ & BG12082 & 31 & $21,8 \cdot 0$ & Transmembrane (4) & SigA and/or others \\
\hline YdbR & BG12085 & 57 & 52 & & SigA and/or others \\
\hline $\mathrm{YdcC}$ & BG12090 & 42 & $28,27,26$ & Transmembrane (1) & SigE \\
\hline $\mathrm{YdcN}$ & BG12101 & 15 & 22 & & NT \\
\hline YdhO & BG12192 & 48 & 45 & Transmembrane $(8)$ & SigA and/or others \\
\hline YerC & BG12831 & 12 & 37 & & SigA and/or others \\
\hline YerQ & $B G 12843$ & 33 & 28 & & NT \\
\hline YfhD & $B G 12879$ & $7 \cdot 3$ & $16,14,11$ & & SigF \\
\hline YfkD & BG12921 & 30 & 29 & Signal sequence & SigG \\
\hline YfmC & BG12954 & 35 & 32 & Signal sequence & SigA and/or others \\
\hline YgaK & BG12231 & 41 & 46,45 & & ND \\
\hline $\mathrm{YhcH}$ & $B G 11586$ & 34 & 31 & & SigA and/or others \\
\hline YhcM & $B G 11591$ & 17 & 22,20 & Signal sequence & SigF \\
\hline YhcV & $B G 11600$ & 15 & 16 & & SigG \\
\hline YhcY & BG13005 & 42 & 44 & & ND \\
\hline YheC & BG13035 & 42 & 42 & & SigE \\
\hline YhfE & BG13051 & 39 & 43 & & SigA and/or others \\
\hline YhfS & BG13063 & 38 & 11 & Transmembrane (1) & SigA and/or others \\
\hline YhjI & $B G 13075$ & 45 & 28 & Transmembrane (12) & SigA and/or others \\
\hline YhjR & BG13084 & 17 & 16,15 & & SigE \\
\hline YhxC & BG11058 & 31 & 37 & & SigE \\
\hline Yis Y & BG13104 & 31 & 30 & & SigG \\
\hline YitS & $B G 12244$ & 31 & 30 & & $\mathrm{ND}$ \\
\hline YjbX & $B G 13153$ & 26 & 43 & & ND \\
\hline $\mathrm{YjcN}$ & $B G 13167$ & 12 & 17 & Signal sequence & NT \\
\hline $\mathrm{YjdH}$ & BG13180 & 15 & $6 \cdot 5,6 \cdot 0,5 \cdot 5$ & Signal sequence & SigE \\
\hline YjfA & $B G 13185$ & 17 & 18 & Signal sequence & SigE \\
\hline YjqC & BG13220 & 31 & 31 & & ND \\
\hline YkzE & BG13333 & $6 \cdot 9$ & 6 & & NT \\
\hline YlaJ & BG13347 & 23 & 25 & Signal sequence & SigG \\
\hline YmaG & $B G 13415$ & 14 & $5 \cdot 0,<4 \cdot 5$ & & SigK \\
\hline YnzH & $B G 13471$ & 12 & 20,19 & & NT \\
\hline YodI & BG13537 & $9 \cdot 1$ & $5 \cdot 0,<4 \cdot 5$ & Transmembrane (1) & NT \\
\hline YopG & $B G 13640$ & $5 \cdot 3$ & 43 & & NT \\
\hline YopK & $B G 13644$ & 45 & 52 & & NT \\
\hline YopY & $B G 13658$ & 11 & 18 & & NT \\
\hline $\mathrm{YqaN}$ & $B G 11265$ & 16 & 21,14 & & NT \\
\hline YqfQ & $B G 11663$ & 26 & 33 & & SigK \\
\hline YqiX & $B G 11727$ & 28 & 29 & & SigA and/or others \\
\hline
\end{tabular}


Table 4. (cont.)

\begin{tabular}{|c|c|c|c|c|c|}
\hline \multirow[t]{2}{*}{ Protein name } & \multirow[t]{2}{*}{ ORF ID } & \multicolumn{2}{|c|}{ Molecular mass ( $\mathrm{kDa})$} & \multirow[t]{2}{*}{ Localization signal } & \multirow[t]{2}{*}{ Possible regulator } \\
\hline & & $\begin{array}{l}\text { Deduced from } \\
\text { sequence }\end{array}$ & $\begin{array}{l}\text { Calculated by } \\
\text { SDS-PAGE }\end{array}$ & & \\
\hline YqjR & $B G 11747$ & 50 & 14 & Signal sequence & ND \\
\hline YrdD & $B G 12284$ & 6 & 14 & & NT \\
\hline YrkC & $B G 11769$ & 21 & 17 & & SigK \\
\hline YsnD & $B G 12335$ & 12 & $5 \cdot 5$ & Signal sequence & SigE \\
\hline YtaA & BG13821 & 41 & 43,42 & & SigK \\
\hline YtcC & $B G 13830$ & 46 & 44 & & SigK \\
\hline YtdI & $B G 13837$ & 30 & 35 & & SigA and/or others \\
\hline YtfJ & $B G 13849$ & 16 & 18 & & SigF \\
\hline YtxO & BG11382 & 17 & 30 & & SigK \\
\hline YufD & $B G 12345$ & 52 & 38 & Transmembrane (14) & NT \\
\hline YuiE & BG13970 & 54 & 59 & Transmembrane (1) & SigA and/or others \\
\hline YusA & $B G 14013$ & 30 & 31 & Signal sequence & SigA and/or others \\
\hline YuxL & BG10463 & 74 & 33 & & SigA and/or others \\
\hline YuzC & BG14052 & 14 & 6 & & SigE \\
\hline YvaB & $B G 14058$ & 23 & 27 & & SigA and/or others \\
\hline YvdD & $B G 12413$ & 21 & 28 & & SigA and/or others \\
\hline YvdO & $B G 12423$ & 35 & $33,32,31$ & & ND \\
\hline YvdP & $B G 12424$ & 50 & $48,46,45,18$ & & SigG \\
\hline YvgV & BG14104 & 25 & 29 & Signal sequence & SigA and/or others \\
\hline YwdL & BG10608 & 20 & 24,23 & Signal sequence & SigE \\
\hline YwqH & BG12512 & 16 & 21,14 & & SigA and/or others \\
\hline YwrJ & $B G 12526$ & 26 & 29 & Transmembrane (1) & SigK \\
\hline YxeE & $B G 11881$ & 15 & $24-6 \cdot 0$ & & SigE \\
\hline YxkC & $B G 12541$ & 23 & 22 & Transmembrane (1) & SigA and/or others \\
\hline YybI & BG10022 & 30 & 28 & & ND \\
\hline
\end{tabular}

ND, No sigma factors can be suggested that are involved in the protein synthesis, because the strains carrying pMutin T3 did not show LacZ activity on sporulation medium; NT, not analysed, because either the strains have not yet been constructed or the genes were essential for vegetative growth.

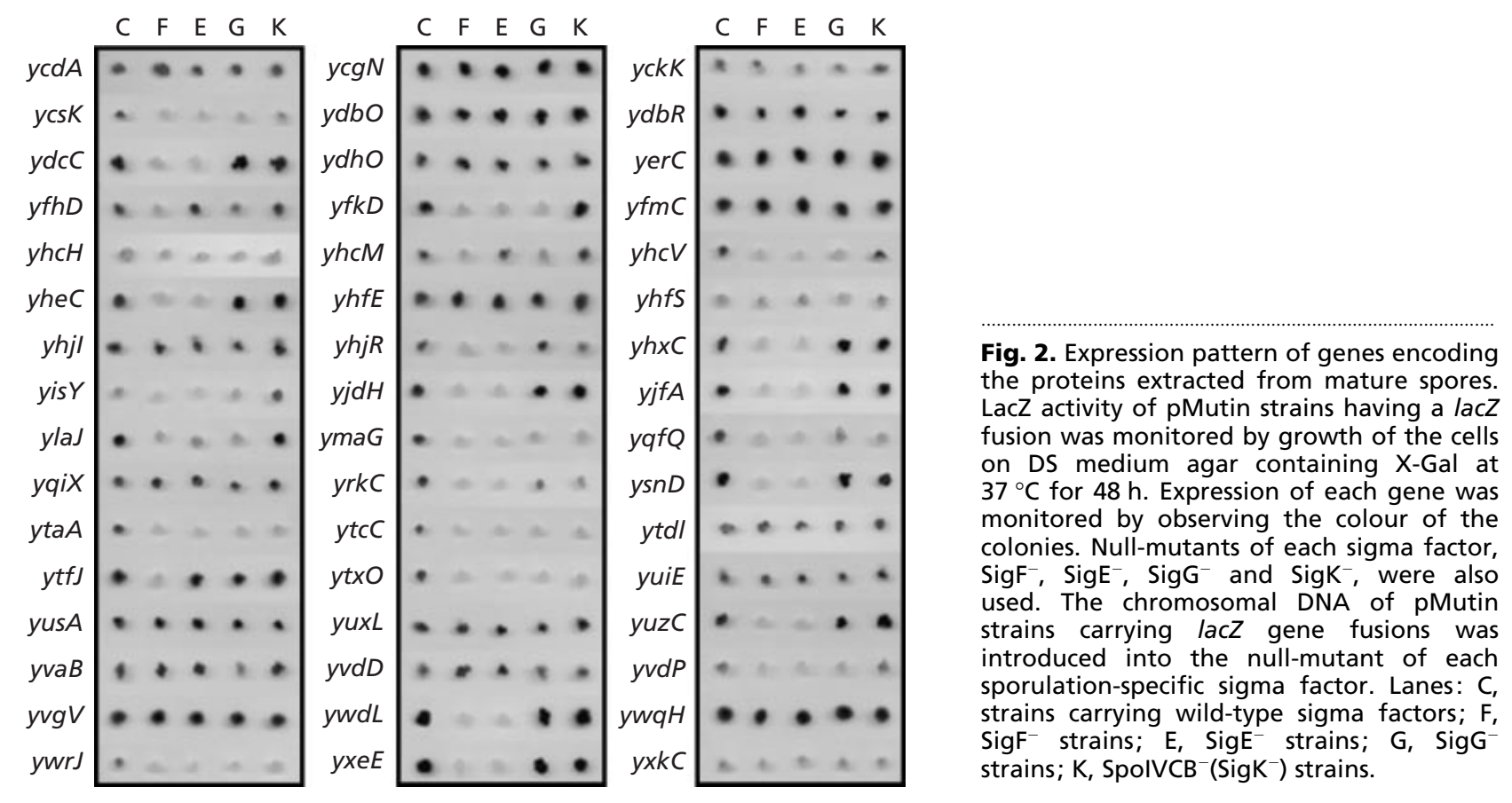


cell compartment is possibly controlled by $\sigma^{\mathrm{K}}$. The expression of the remaining 22 genes is independent of these sigma factors. Fawcett et al. (2000) have found that the transcription of $y h f S, y t f J$ and $y w d L$ is reduced in mutant cells of spoOA or sigF using DNA arrays.

\section{DISCUSSION}

\section{Spore proteins of $B$. subtilis}

Bacterial spores contain unique high- and lowmolecular-mass proteins. Some of these proteins contribute directly or indirectly to the unique characteristics of spores, such as dormancy, a high degree of resistance and particularly a unique cell morphology. Therefore, a comprehensive analysis of protein composition will provide useful basic information and will facilitate our understanding of the cell differentiation of $B$. subtilis at the molecular level. Previous studies have reported that at least 45 proteins are associated with spores of $B$. subtilis (Driks, 1999; Henriques \& Moran, 2000). These studies have all been performed by resolution and detection of the spore proteins using one-dimensional gel electrophoresis. Identification of spore proteins has technical problems, because both the extraction of total proteins from mature spores and the purification of each protein are quite difficult. We applied a recently developed LC-MS/MS system to this work and succeeded in identifying over 200 proteins and polypeptides from B. subtilis spores (Fig. 1; Table 2). It is notable that this system can distinguish each protein in a protein mixture and identify unknown polypeptides; it can also detect many membrane-bound proteins. These observations indicate that this system is usable for proteome analyses of bacterial spores.

The majority of known spore proteins, small acidsoluble proteins (SASPs) and coat proteins of B. subtilis were identified in this work. Some of these proteins are presumed to function in the morphogenesis and germination of spores, as described previously (Piggot \& Losick, 2001; Paidhungat \& Setlow, 2001). A total of 16 polypeptides originally characterized as spore-coat proteins were identified under our experimental conditions. In this work, $\operatorname{CotJB}$, the coat protein encoded by the second gene of the cotJ operon (Seyler $e t$ al., 1997; Henriques et al., 1995), was detected in gel slice no. 64, while this protein had not been isolated from spores in previous studies. Four coat proteins, CotM (Henriques et al., 1997), CotT (Bourne et al., 1991), CotV (Zhang et al., 1993) and CotX (Zhang et al., 1993), were not detected in this work. TasA has been reported as the protein associated with spores at least under certain conditions (Serrano et al., 1999). It was also not found in this study. Other coat proteins, $\operatorname{Cot} B, \operatorname{Cot} C$, $\operatorname{Cot} \mathrm{D}, \operatorname{Cot} \mathrm{E}, \mathrm{CotS}$ and $\operatorname{Cot} \mathrm{Y}$, were dispersed among several gel slices. These results suggest that some spore proteins are subjected to processing or cross-linking during sporulation. Indeed, we have previously identified one of the sporulation-specific proteases, YabG, which is involved in processing of some coat proteins during morphogenesis of the coat layer (Takamatsu et al., 2000), and this protein was found in gel slice no. 57. SodA and Tgl were found in gel slices nos 33 and 29, respectively; these are possibly involved in protein modification (Henriques et al., 1998; Kobayashi et al., 1998). Other processing enzymes, AprX (Valbuzzi et al., 1999), FtsH (Deuerling et al., 1995) and Mpr (Rufo et al., 1990; Sloma et al., 1990), were also identified from spores in this work. We speculate that these and some unidentified modification enzymes are involved in sporulation or germination processes, either directly or indirectly. Seventeen genes deduced to encode SASPs were found in the genome sequence project (Kunst et al., 1997) and many of the corresponding polypeptides have been identified from acid extracts of spores (Francesconi et al., 1988; Bagyan et al., 1998). In this work, seven of them were identified from spores extracted in the absence of acidic reagents. We prepared spore samples very carefully, as described Methods. In this study, we did not use any spore preparations that contained a protein, Hag (flagellin), which would suggest contamination by vegetative cells. Hag is a major component of flagella, which is associated with vegetative cells and is involved in the motility of B. subtilis (LaVallie \& Stahl, 1989; Hirose et al., 2000).

Here, we identified not only spore-specific proteins but also proteins involved in DNA maintenance, protein synthesis, metabolism, transport, secretion, proteolysis, etc. (Table 3 ). These proteins are probably required for resistance, germination or outgrowth of spores. It is, of course, difficult to solubilize total proteins from dormant spores even with harsh treatments. The above results indicate that at least some proteins organized in the spore coat, cortex and core are obtained under our experimental conditions. Many functionally unknown genes remain in the B. subtilis database and some of them could encode spore proteins.

\section{Similarity of the spore proteins}

Analysis of amino acid sequences showed the similarity of primary sequence between novel proteins and previously known spore proteins. The entire sequence of $\mathrm{YnzH}$ was similar to the corresponding region of $\operatorname{CotC}$ $(71 \%)$. YtaA is a paralogue of CotS, and YtcC is a paralogue of CotSA (YtxN). The cotS operon is located near both $y t a A$ and $y t c C$. cotS, $\operatorname{cotSA}(y \operatorname{txN}), y t a A$ and $y t c C$ are transcribed under the regulation of $\sigma^{\mathrm{K}}$. Therefore, we hypothesize that the functions of CotS and $\mathrm{YtaA}$ and those of CotSA (YtxN) and $\mathrm{YtcC}$ are similar. $\mathrm{YjqC}$ has primary sequence similarity $(31 \%)$ to the corresponding region of CotJC. Both YhxC and YxbG, whose functions are still unknown, are similar to glucose dehydrogenase (GDH). The C-terminal half of YrvJ has similarity $(28 \%)$ to the corresponding region of CwlD. Although we obtained important information from comparisons of the amino acid sequences, the function of some novel spore proteins is still unknown. Our preliminary results showed that the phenotypes of some of the pMutin strains, such as the heat resistance of 
spores, are the same as the wild-type, but others are defective in germination (H. Takamatsu, R. Kuwana, M. Fujibayashi \& K. Watabe, unpublished data).

\section{Deduced localization of novel spore proteins}

By analysis of gene expression using lacZ fusions, we found that the transcription of 26 genes encoding novel spore proteins was controlled by sporulation-specific sigma factors, namely $\sigma^{\mathrm{F}}, \sigma^{\mathrm{E}}, \sigma^{\mathrm{G}}$ and $\sigma^{\mathrm{K}}$. These sigma factors are temporally and spatially activated and regulate gene expression in a compartment-specific fashion (Igo \& Losick, 1986; Stragier \& Losick, 1996).

In general, spores of $B$. subtilis have three distinct structures, the core, the cortex and the coat. The proteins synthesized in the mother-cell compartment under the regulation of $\sigma^{\mathrm{E}}$ and $\sigma^{\mathrm{K}}$ will be localized in the cortex or coat. In contrast, proteins synthesized in the forespore under the regulation of $\sigma^{\mathrm{F}}$ and $\sigma^{\mathrm{G}}$ will be localized in the cortex and core (Driks, 1999). Additionally, TasA has been reported as a protein that is synthesized under the regulation of $\sigma^{\mathrm{H}}$, associated with the spore coat and involved in spore morphogenesis (Serrano et al., 1999). A core protein synthesized in the mother-cell compartment or a coat protein synthesized in the forespore has never been reported. Possible signal sequences or transmembrane regions were found in many proteins, and these may facilitate the translocation of the proteins to outer- and inner-spore membranes. From these observations, we have predicted the site of localization of newly identified proteins. YcsK, YheC, YhjR, YhxC, YmaG, YqfQ, YrkC, YtaA, YtcC, YtxO, YuzC and YxeE are possibly synthesized in the mother-cell compartment and assemble on the spore coat. YdcC, YjdH, YjfA, YsnD, YwdL and YwrJ are also possibly synthesized in the mother-cell compartment. We speculate that they localize to the outer-spore membrane or cortex because they have a deduced signal sequence or transmembrane sequence(s). This speculation is supported by the following facts. Both DacB and DacF are cortex proteins and they have a typical signal sequence (Buchanan \& Ling, 1992; Wu et al., 1992). DacB is controlled by $\sigma^{\mathrm{E}}$ (Simpson et al., 1994) and DacF is controlled by $\sigma^{\mathrm{F}}$ (Schuch \& Piggot, 1994). SleB and YrbB also have a signal sequence and their localization in the cortex has been shown by immunological studies (Moriyama et al., 1999; Takamatsu et al., 1998). DacB, $\mathrm{DacF}$, SleB and YrbB were all detectable in our analysis (Table 2). YfhD, YhcV, YisY, YtfJ and YvdP are possibly synthesized in the forespore and exist in the core. YfkD, YhcM and YlaJ have a deduced signal sequence. These proteins are possibly synthesized in the forespore and localize to the inner-spore membrane or cortex. To understand the precise localization site of the newly identified spore proteins and their functions in the cell, we are currently performing a comprehensive analysis of these proteins by using a combination of SDS-PAGE and LC-MS/MS.

\section{ACKNOWLEDGEMENTS}

We thank the Japanese and European Consortia for Functional Analysis of the B. subtilis Genome for providing the pMutin strains. This work was supported by Grant-in-aids for Scientific Research on Priority Areas (C) 'Genome Biology' from the Ministry of Education, Culture, Sports, Science and Technology of Japan.

\section{REFERENCES}

Aronson, A. I. \& Fitz-James, P. (1976). Structure and morphogenesis of the bacterial spore coat. Bacteriol Rev 40, 360-402.

Bagyan, I., Setlow, B. \& Setlow, P. (1998). New small, acid-soluble proteins unique to spores of Bacillus subtilis: identification of the coding genes and regulation and function of two of these genes. J Bacteriol 180, 6704-6712.

Bourne, N., Fitz-James, P. C. \& Aronson, A. I. (1991). Structural and germination defects of Bacillus subtilis spores with altered contents of a spore coat protein. J Bacteriol 173, 6618-6625.

Buchanan, C. E. \& Ling, M.-L. (1992). Isolation and sequence analysis of $d a c B$, which encodes a sporulation-specific penicillinbinding protein in Bacillus subtilis. J Bacteriol 174, 1717-1725.

Cutting, S. M. \& Vander Horn, P. B. (1990). Genetic analysis. In Molecular Biological Methods for Bacillus, pp. 27-64. Edited by C. R. Harwood \& S. M. Cutting. Chichester: Wiley.

Deuerling, E., Paeslack, B. \& Schumann, W. (1995). The $f t s H$ gene of Bacillus subtilis is transiently induced after osmotic and temperature upshift. J Bacteriol 177, 4105-4112.

Driks, A. (1999). Bacillus subtilis spore coat. Microbiol Mol Biol Rev 63, 1-20.

Driks, A. (2001). Proteins of the spore core and coat. In Bacillus subtilis and its Closest Relatives from Genes to Cells, pp. 527-535. Edited by A. L. Sonenshein, J. A. Hoch \& R. Losick. Washington, DC: American Society for Microbiology.

Fawcett, P., Eichenberger, P., Losick, R. \& Youngman, P. (2000). The transcriptional profile of early to middle sporulation in Bacillus subtilis. Proc Natl Acad Sci U S A 97, 8063-8068.

Francesconi, S. C., MacAlister, T. J., Setlow, B. \& Setlow, P. (1988). Immunoelectron microscopic localization of small, acid-soluble spore proteins in sporulating cells of Bacillus subtilis. J Bacteriol 170, 5963-5967.

Goldman, R. C. \& Tipper, D. J. (1978). Bacillus subtilis spore coats: complexity and purification of a unique polypeptide component. J Bacteriol 135, 1091-1106.

Gould, G. W. (1983). Mechanisms of resistance and dormancy. In The Bacterial Spores, vol. 2, pp. 173-210. Edited by A. Hurst \& G. W. Gould. London: Academic Press.

Helmann, J. D. \& Moran, C. P., Jr (2001). RNA polymerase and sigma factors. In Bacillus subtilis and its Closest Relatives from Genes to Cells, pp. 527-535. Edited by A. L. Sonenshein, J. A. Hoch \& R. Losick. Washington, DC: American Society for Microbiology.

Henner, D. J. (1990). Inducible expression of regulatory genes in Bacillus subtilis. Methods Enzymol 185, 223-228.

Henriques, A. O \& Moran, C. P., Jr (2000). Structure and assembly of the bacterial endospore coat. Methods 20, 95-110.

Henriques, A. O., Beall, B. W., Roland, K. \& Moran, C. P., Jr (1995). Characterization of $\cot$, a $\sigma^{\mathrm{E}}$-controlled operon affecting the polypeptide composition of the coat of Bacillus subtilis spores. J Bacteriol 177, 3394-3406.

Henriques, A. O., Beall, B. W. \& Moran, C. P., Jr (1997). CotM of 
Bacillus subtilis, a member of the $\alpha$-crystallin family of stress proteins, is induced during development and participates in spore outer coat formation. J Bacteriol 179, 1887-1897.

Henriques, A. O., Melsen, L. R. \& Moran, C. P., Jr (1998). Involvement of superoxide dismutase in spore coat assembly in Bacillus subtilis. J Bacteriol 180, 2285-2291.

Hirose, I., Sano, K., Shioda, I., Kumano, M., Nakamura, K. \& Yamane, K. (2000). Proteome analysis of Bacillus subtilis extracellular proteins: a two-dimensional protein electrophoretic study. Microbiology 146, 65-75.

Igo, M. M. \& Losick, R. (1986). Regulation of a promoter that is utilized by minor forms of RNA polymerase holoenzyme in Bacillus subtilis. J Mol Biol 191, 615-624.

Kobayashi, K., Hashiguchi, K., Yokozeki, K. \& Yamanaka, S. (1998). Molecular cloning of the transglutaminase gene from Bacillus subtilis and its expression in Escherichia coli. Biosci Biotechnol Biochem 62, 1109-1114.

Kunst, F., Ogasawara, N., Moszer, I. \& 148 other authors (1997). The complete genome sequence of the gram-positive bacterium Bacillus subtilis. Nature 390, 249-256.

LaVallie, E. R. \& Stahl, M. L. (1989). Cloning of the flagellin gene from Bacillus subtilis and complementation studies of an in vitroderived deletion mutation. J Bacteriol 171, 3085-3094.

Moriyama, R., Fukuoka, H., Miyata, S., Kudoh, S., Hattori, A., Kozuka, S., Yasuda, Y., Tochikubo, K. \& Makino, S. (1999). Expression of a germination-specific amidase, SleB, of bacilli in the forespore compartment of sporulating cells and its localization on the exterior side of the cortex in dormant spores. J Bacteriol 181, 2373-2378.

Paidhungat, M. \& Setlow, P. (2001). Spore germination and outgrowth. In Bacillus subtilis and its Closest Relatives from Genes To Cells, pp. 537-548. Edited by A. L. Sonenshein, J. A. Hoch \& R. Losick. Washington, DC: American Society for Microbiology.

Piggot, P. J. \& Losick, R. (2001). Sporulation genes and intercompartmental regulation. In Bacillus subtilis and its Closest Relatives from Genes To Cells, pp. 483-517. Edited by A. L. Sonenshein, J. A. Hoch \& R. Losick. Washington, DC: American Society for Microbiology.

Rufo, G. A., Jr, Sullivan, B. J., Sloma, A. \& Pero, J. (1990). Isolation and characterization of a novel extracellular metalloprotease from Bacillus subtilis. J Bacteriol 172, 1019-1023.

Sambrook, J., Fritsch, E. F. \& Maniatis, T. (1989). Molecular Cloning: a Laboratory Manual, 2nd edn. Cold Spring Harbor, NY: Cold Spring Harbor Laboratory.

Schuch, R. \& Piggot, P. J. (1994). The dacF-spoIIA operon of Bacillus subtilis, encoding $\sigma^{\mathrm{F}}$, is autoregulated. J Bacteriol 176, 4104-4110.

Serrano, M., Zilhao, R., Ricca, E., Ozin, A. J., Moran, C. P., Jr \& Henriqous, A. O. (1999). A Bacillus subtilis secreted protein with a role in endospore coat assembly and function. J Bacteriol 181, 3632-3643.

Seyler, R. W., Jr, Henriques, A. O., Ozin, A. J. \& Moran, C. P., Jr (1997). Assembly and interactions of cotJ-encoded proteins, constituents of the inner layers of the Bacillus subtilis spore coat. Mol Microbiol 25, 955-966.

Shevchenko, A., Wilm, M., Vorm, O. \& Mann, M. (1996). Mass spectrometric sequencing of proteins silver-stained polyacrylamide gels. Anal Chem 68, 850-858.

Simpson, E. B., Hancock, T. W. \& Buchanan, C. E. (1994). Transcriptional control of $d a c B$, which encodes a major sporulationspecific penicillin-binding protein. J Bacteriol 176, 7767-7769.

Sloma, A., Rudolph, C. F., Rufo, G. A., Jr, Sullivan, B. J., Theriault, K. A., Ally, D. \& Pero, J. (1990). Gene encoding a novel extracellular metalloprotease in Bacillus subtilis. J Bacteriol 172, 1024-1029.

Stragier, P. \& Losick, R. (1996). Molecular genetics of sporulation in Bacillus subtilis. Annu Rev Genet 30, 297-341.

Takamatsu, H., Hiraoka, T., Kodama, T., Koide, H., Kozuka, S., Tochikubo, K. \& Watabe, K. (1998). Cloning of a novel gene $y r b B$, encoding a protein located in the spore integument of Bacillus subtilis. FEMS Microbiol Lett 166, 361-367.

Takamatsu, H., Kodama, T., Imamura, A., Asai, K., Kobayashi, K., Nakayama, T., Ogasawara, N. \& Watabe, K. (2000). The Bacillus subtilis yabG gene is transcribed by SigK RNA polymerase during sporulation, and yabG mutant spores have altered coat protein composition. J Bacteriol 182, 1883-1888.

Vagner, V., Dervyn, E. \& Ehrlich, S. D. (1998). A vector for systematic gene inactivation in Bacillus subtilis. Microbiology 144, 3097-3104.

Valbuzzi, A., Ferrari, E. \& Albertini, A. M. (1999). A novel member of the subtilisin-like protease family from Bacillus subtilis. Microbiology 145, 3121-3127.

Wu, J.-J., Schuch, R. \& Piggot, P. J. (1992). Characterization of a Bacillus subtilis sporulation operon that includes genes for an RNA polymerase $\sigma$ factor and for a putative DD-carboxypeptidase. J Bacteriol 174, 4885-4892.

Yates, J. R., 3rd, Eng, J. K., McCormack, A. L. \& Schieltz, D. (1995). Method to correlate tandem mass spectra of modified peptides to amino acid sequences in the protein database. Anal Chem 67, $1426-1436$.

Zhang, J., Fitz-James, P. C. \& Aronson, A. I. (1993). Cloning and characterization of a cluster of genes encoding polypeptides present in the insoluble fraction of the spore coat of Bacillus subtilis. J Bacteriol 175, 3757-3766.

Received 30 May 2002; revised 7 August 2002; accepted 12 September 2002. 